engt, dann mit $10 \mathrm{~cm}^{3}$ Petroläther versetzt und auf $-80^{\circ}$ abgekühlt. Dabei schied sich aus der roten Lösung eine gelb-braune Substanz ab, welche in $1 \mathrm{~cm}^{3}$ Benzol gelöst und nochmals bei $-80^{\circ}$ mit Petroläther gefällt wurde. Zur Reinigung von $\mathrm{P}\left(\mathrm{C}_{6} \mathrm{H}_{5}\right)_{3}$ mußte die ausgefallene gelbe Substanz 4-mal mit $15 \mathrm{~cm}^{3}$ konz. $\mathrm{HCl}$ durchgerührt, getrocknet, mit $15 \mathrm{~cm}^{3}$ Heptan gewaschen und nochmals getrocknet werden. Ausbeute $130 \mathrm{mg}$ (25\%). Zur weiteren Reinigung wurde die Substanz im Hochvakuum bei $180^{\circ}$ sublimiert.

Analyse von $\mathrm{C}_{5} \mathrm{H}_{5} \mathrm{Mn}(\mathrm{CO})_{2} \mathrm{P}\left(\mathrm{C}_{6} \mathrm{H}_{5}\right)_{3} \quad(438,5)$

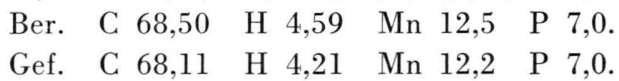

Die schwachgelbliche Substanz schmilzt bei $216-218^{\circ}$ (K of le r-Bank) und läßt sich im Hochvakuum bei $180^{\circ}$ langsam sublimieren. Sie ist an der Luft beständig, unlöslich in heißem Wasser und konz. HCl, schwer löslich in Aceton, Methanol und Äther, löslich in Toluol, $\mathrm{CCl}_{4}$, Benzol und Tetrahydrofuran. Die Lösungen zersetzen sich langsam an der Luft.

Cyclopentadienylmang ancarbonyl-ditriphenylphos phin. Analog zur Darstellung der monosubstituierten Verbindung wurden $104 \mathrm{mg}(0,51$ $\mathrm{mMol}) \quad \mathrm{C}_{5} \mathrm{H}_{5} \mathrm{Mn}(\mathrm{CO})_{3}$ und $1150 \mathrm{mg}$ (4,4 mMole) $\mathrm{P}\left(\mathrm{C}_{6} \mathrm{H}_{5}\right)_{3}$, gelöst in $25 \mathrm{~cm}^{3}$ Benzol bis zur Abspaltung von 1,47 mMolen (218\%) CO bestrahlt. Die rote, durch Zersetzungsprodukte getrübte Lösung wurde filtriert und am Rotationsverdampfer das Lösungsmittel abge- zogen. Nach Aufnehmen der zurückgebliebenen roten schmierigen Substanz in $5 \mathrm{~cm}^{3}$ Petroläther und Abkühlen der Lösung auf $-80^{\circ}$ fiel eine braun-rote Substanz aus, welche 4-mal mit $15 \mathrm{~cm}^{3}$ konz. $\mathrm{HCl}$ durchgerührt, dann getrocknet und 5-mal mit $20 \mathrm{~cm}^{3}$ Methanol gewaschen wurde. Ausbeute $167 \mathrm{mg}$ (48\%).

Analyse von $\mathrm{C}_{5} \mathrm{H}_{5} \mathrm{Mn}(\mathrm{CO})\left[\mathrm{P}\left(\mathrm{C}_{6} \mathrm{H}_{5}\right)_{3}\right]_{2} \quad(672,6)$

Ber. C 74,99 H 5,24 Mn 8,2 P 9,2.

Gef. C 74,90 H 5,33 Mn 8,3 P 9,0.

Die orange gefärbte Substanz hat keinen Schmelzpunkt und zersetzt sich bei $\sim 110^{\circ}$. Sie ist nicht sublimierbar, unlöslich in Methanol, heißem Wasser und konz. HCl, schwer löslich in Äther und Aceton, löslich in Toluol, Benzol, $\mathrm{CCl}_{4}$ und Tetrahydrofuran. Die Lösungen zersetzen sich an der Luft.

Versuche zur thermischen Bildung der Triphenylphosphinderivate

a) $765 \mathrm{mg}(3,75 \mathrm{mMole}) \mathrm{C}_{5} \mathrm{H}_{5} \mathrm{Mn}(\mathrm{CO})_{3}$ und $1860 \mathrm{~g}$ (7,0 mMole) $\mathrm{P}\left(\mathrm{C}_{6} \mathrm{H}_{5}\right)_{3}$ wurden mit $30 \mathrm{~cm}^{3}$ Dekan 20 Stdn. am Rückfluß gekocht.

b) $204 \mathrm{mg}(1,0 \mathrm{mMol}) \mathrm{C}_{5} \mathrm{H}_{5} \mathrm{Mn}(\mathrm{CO})_{3}$ und $1100 \mathrm{mg}$ $\mathrm{P}\left(\mathrm{C}_{6} \mathrm{H}_{5}\right)_{3} \quad(4,2 \mathrm{mMole})$ wurden im abgeschmolzenen Rohr 20 Stdn. auf $200^{\circ}$ erhitzt.

In beiden Fällen konnte keine Reaktion zwischen $\mathrm{C}_{5} \mathrm{H}_{5} \mathrm{Mn}(\mathrm{CO})_{3}$ und $\mathrm{P}\left(\mathrm{C}_{6} \mathrm{H}_{5}\right)_{3}$ nachgewiesen werden.

Wir danken der Deutschen Forschungsgemeinschaf und dem Fonds der Chemischen Industrie für die finanzielle Unterstützung der vorliegenden Arbeit und der Ethyl-Corp or a tion (Detroit) für das zur Verfügung gestellte Cyclopentadienylmangantricarbonyl.

\section{Umsetzung von Alkylschwefeltrichlorid und Schwefeltetrachlorid mit Äthylmercaptan}

\author{
Von F. FehÉr und K.-H. SCHÄFER ${ }^{2}$
}

Institut für Anorganische Chemie der Universität Köln

(Z. Naturforschg. 17 b, 849 [1962] ; eingegangen am 18. September 1962)

Die Reaktion zwischen Alkylschwefeltrichlorid bzw. Schwefeltetrachlorid und Äthylmercaptan wurde auf zwei verschiedenen Wegen durchgeführt. Entweder wurde das Chlorid vorgelegt und das Mercaptan zugetropft oder das Mercaptan wurde in einer gekühlten ätherischen Lösung vorgelegt und das in Äther suspendierte Chlorid in größeren Anteilen zugefügt.

Die Wärmetönung der Reaktion ist positiv. Beim Zutropfen des Mercaptans zu dem in Äther suspendierten Alkylschwefeltrichlorid wurde ein von der Tropfgeschwindigkeit abhängiger starker Temperaturanstieg der Reaktionslösung beobachtet. Das Ende der Umsetzung wurde durch einen plötzlichen Abfall der Temperatur angezeigt. Diese Indikation war so scharf, daß auf 1 Mol des eingesetzten Chlorids auch genau 3 bzw. 4 Mole Äthylmercaptan verbraucht wurden. Die Temperaturen, bei denen die Umsetzungen zur Durchführung gelangten, wurden von Versuch zu Versuch zwi-

1 64. Mitt.: F. FehÉr, K.-H. Schäfer u. W. Becher, Z. Naturforschg. 17 b, 847 [1962]. schen -20 und $-70{ }^{\circ} \mathrm{C}$ variiert, um Primärprodukte der Reaktion zu fassen.

Im umgekehrten Falle wurde das zwei- bis fünffache der erforderlichen Menge Äthylmercaptan in $-70^{\circ}$ kaltem Äther vorgelegt und die ebenso kalte ätherische Suspension des Chlorids portionsweise zugegeben.

Nach Aufarbeitung der Reaktionsmischungen wurde die Bildung von

1. Methyläthyldisulfan und Diäthyldisulfan im Verhältnis etwa $1: 1$ festgestellt, so daß folgende Bruttogleichung gilt :

$$
\begin{aligned}
\mathrm{R}-\mathrm{SCl}_{3}+3 \mathrm{C}_{2} \mathrm{H}_{5} \mathrm{SH} & \\
& =\mathrm{R}-\mathrm{S}_{2}-\mathrm{C}_{2} \mathrm{H}_{5}+\mathrm{C}_{2} \mathrm{H}_{5}-\mathrm{S}_{2}-\mathrm{C}_{2} \mathrm{H}_{5}+3 \mathrm{HCl} .
\end{aligned}
$$

2. Bei Umsetzung von $\mathrm{SCl}_{4}$ mit $\mathrm{C}_{2} \mathrm{H}_{5} \mathrm{SH}$ konnte Diäthyldisulfan und Diäthyltrisulfan im Verhältnis $1: 1$ isoliert werden:

$$
\mathrm{SCl}_{4}+4 \mathrm{C}_{2} \mathrm{H}_{5} \mathrm{SH}=\left(\mathrm{C}_{2} \mathrm{H}_{5}\right)_{2} \mathrm{~S}_{2}+\left(\mathrm{C}_{2} \mathrm{H}_{5}\right)_{2} \mathrm{~S}_{3}+4 \mathrm{HCl} .
$$

Die primäre Bildung von Verbindungen mit verzweigter Schwefelkette konnte also auch unter mildesten Bedingungen in keinem Falle beobachtet werden. Ramanspektroskopische Untersuchungen der bei tiefen Temperaturen isolierten und zwischen -30 und $-40{ }^{\circ} \mathrm{C}$ registrierten Reaktionsprodukte zeigten dementsprechend nur die Frequenzen der nach den oben angegebenen Reaktionsgleichungen gebildeten Disulfane und des Diäthyltrisulfans.

2 K. H. Schärer, Inaugural-Dissertation, Universität Köln 1961. 